
\title{
25 Research Soure \\ Experiences of Nurses on Perceived Therapeutic Communication Barriers in Ethiopia, A Qualitative Study
}

SOLOMON EMISHAW

Bahir Dar University

WORKU ANIMAW

Bahir Dar University

TIGABU DESIE

Debre Tabor University

NIGUSIE SELOMON

Debre Tabor University

CHALIE MAREW

Debre Tabor University

ADANE BIRHANU

Debre Tabor University

AGIMASIE TIGABU

Debre Tabor University

MOGES WUBNEH ( $\square$ wmoges7@gmail.com )

Debre Tabor University

\section{Research Article}

Keywords: Explore, communication, barriers, nurse, qualitative, Ethiopia

Posted Date: March 9th, 2021

DOl: https://doi.org/10.21203/rs.3.rs-244749/v1

License: (1) (1) This work is licensed under a Creative Commons Attribution 4.0 International License. Read Full License 


\section{Abstract}

Background: Communication is the exchanging of the message by using verbal and non-verbal ways. It is an essential skill, particularly for nurses. Communication barriers are hinders that affect the giving and receiving of information between nurses and patients.

Objective: The purpose of this study was to explore the experience of nurses on perceived communication barriers.

Methods: An exploratory study design was employed using a qualitative approach. A purposive sampling technique was used to select 15 nurses who were interviewed both in focused group discussion and indepth interview using an unstructured interview guide. Interviews were audio-taped, transcribed verbatim and analyzed using thematic analysis.

Results: Perceived communication barriers were explored and the analysis was made using deductive approach thematic analysis. Perceived communication barriers were categorized into five themes includes; socio-demographic related communication, common related communication, nurse related communication, patient-related communication, and environment-related communication barriers.

Conclusion: Nurses, patients, and the environments are the main perceived communication barriers as indicated in both in-depth interviews and focused group discussion. Lack of medical facilities or access is the main barrier of the nurse to patient communication, which needs great attention of the stakeholders. To enhance communication with the patients; nurses and other stakeholders like the ministry of health, health bureaus, and hospital authorities need to recognize the communication barriers. Giving awareness on the communication barriers for the nurses helps to minimize the barriers and improve the nurses to patients' communication.

\section{Introduction}

Communication is the process of sharing an idea in verbal and non-verbal ways. It essential skill for nurses since nurses take much time with clients and families than any other health workers. When nurses communicate properly with their clients; the clients are eager to report their problem to their nurses (1).

Therapeutic communication is vital for better care for patients (2). It can facilitate greater adherence of the patients to treatment options and helps to support families (3). Effective therapeutic communication has become increasingly reported as a key component in better nursing care outcome (4). It is a multidimensional and dynamic process that takes place in the hospital or related places (5).

Communication barriers are obstacles which hide the exchanging of idea between two or more persons. Speech barriers happen when the person can not speak similar speech or language or do not have an identical understanding capacity. This difference leads to an unable to share the message which results in diagnosis and treatment errors. Even within a similar speech, there are dialect differences. It is also 
affected by time constraints, lack of communication skills, and environmental factors which cause poor patient outcomes $(4,6-9)$.

The studies in Manchester, England, and Canada indicated that poor communication between patients and the nurses result in an increased length of stay, wastage of the resource, patient dissatisfaction, absence of confidence, and frustration for both the nurses and the patients $(1,10)$. Failure to recognize the two-way communication capability quite often leads to negative conclusions and attitudes (2).

Research findings from the United States, Australia, Norway, and Brazil have confirmed that there were problems on the patients' side as a result of inadequate time given for them during nurse-patient engagement which in turn limits patients' access to communicate their informational needs. Nurses do not understand communication as a key element to nursing care that should be used, especially at the time of the patient admission represented by feelings of fear, insecurity, and anxiety $(8,11-13)$. Evidence in Iran, Saudi Arabia, and Ghana showed that the patient, nurse, and environmental related issues affect the communication between nurses and patients which have the ultimate result in reducing communication $(4,9,14)$.

In Ethiopia, there is no study found which shows the experiences of nurses on perceived communication barriers qualitatively. So the main reason to conduct this study was to assess the experiences of nurses on perceived communication barriers. Study findings will be used as input for decision-makers and responsible bodies like the federal ministry of health $(\mathrm{FMOH})$, regional health bureaus, academic institutions, hospital authorities, and nurses, which help to decide what needs to be done to improve nurses to patients' communications. It will be used as baseline data for the researcher who needs to conduct on the area of the nurse to patient communication.

\section{Methods}

\section{Study area, study design and sample size}

The study was conducted in Felege Hiwot comprehensive specialized hospitals (FHCSH) which is found in Bahir Dar City, the capital city of Amhara regional state. It serves about 20 million populations and there were 434 nurses in the hospital. The study employed an exploratory based design which followed a qualitative approach to explore nurses' experiences on the perceived communication barriers. Purposive sampling was used to select the participants. The sample size was considered until data saturation, and seven nurses have participated in an in-depth interview with data saturation was gained at the fourth participant and eight nurses were participated in focused group discussion. Data saturation is a matter of identifying redundancy in the data or relates to the degree to which new data repeat what was expressed in previous data during data collection (15).

\section{Data collection tool and procedure}


An in-depth interview guide unstructured questionnaire was used to elicit information concerned with perceived communication barriers from the nurses' point of view. Detailed information about nurses' thoughts was explored in-depth which was offered a more complete picture of nurses experiences on perceived barriers of the nurse to patient communication.

\section{Data quality assurance}

The accuracy of the transcripts was checked by repetitive listen to the audiotape and by reading the transcripts. For the qualitative study field note and audio, the record was taken. Each interview and focused group discussion was transcribed by cross-checked both the audio record and the field note. The analysis was carried out by using deductive approach thematic analysis which involves coming to the data with some preconceived themes that expect to find reflected there, based on theory or existing knowledge (16). Based on this data were thematized in five major themes. The themes included; sociodemographic related communication barrier with sub-themes of age, sex, religion and marital status, common-related communication barriers with sub-themes of language difference; nurse-related barriers with sub-themes of lack of communication skill, shortage of nurses and workload; patient-related barriers with sub-themes pain, and family interferences; environmental or health setting-related barriers with subthemes of lack of medical facilities and lack of continuous training, inappropriate and busy environment.

\section{Ethical clearance}

Ethical issues within the study were taken into consideration when carried out the study. Ethical clearance was obtained from the institutional review board of Bahir Dar University, college of medicine, and health sciences. Informed consent was obtained from respondents and assured that their participation will be recorded anonymously, and confidentiality of response was maintained throughout the study.

\section{Results}

\section{Socio-demographic characteristics of nurses}

Seven nurses have participated in the in-depth interviews and eight in focused group discussion. Of which $9(60 \%)$ of them were male. The age of the participants ranged from $26-50$ with a mean age of 37.13 years. More than $73 \%$ of the participants were orthodox religion followers.

\section{Socio-demographic related barriers}

Those are barriers that arise in both nurses and patients socio-demographic characteristics. It includes; age, sex, religion and marital status. 
"Age of the nurses also affect communication. Elder nurses refuse the questions of the patient due to physical fatigue while younger nurses are not interesting to care for elder patients (INT 2, 3 and FGD)." As the patients get aged they easily forget what we have told them. For example old age patients with stroke, hypertension, and diabetes mellitus after we taught them, forget everything. Then we lost energy for recurrent taught, and finally, our communication with them was decreased (INT 4 and 6). "It is difficult to communicate with old patients. They cannot

understand easily what we want to communicate with them (FGD)." "When the patients age greater than 60 years old they cannot understand what the nurses said; especially the young nurses are fast, and they cannot understand the patients' age condition (INT 5)." "It is difficult to communicate with old patients. As their age increases, they cannot easily understand us. For example when I was care patients with hypertension who develops stroke. He could not understand what I told. Finally, he said I longer live with this disease, now this may be God want to punish me. One side of my arm and leg is paralyzed; God wants to take me, so whatever you told me I cannot hear you (INT 7)."

Sex is another challenge for the nurse to patient communication. Some patients need the same sex to care for them while others show interest in the opposite sex. This happens especially at a younger age. For example, at one time the elder nurse goes to give care to young patients. She was not interested in care by the elder nurses rather she calls another young nurse that she knows before. What we did during this time was to change the elder one by the young (INT 7). "Female patients prefer male nurses to care for them while male prefer female nurses especially the young ages. This makes disrupted communication between nurses and patients (INT 4, 5 and FGD)."

"Religion is one barrier to communication. For example, at one time I was doing the nursing process of my patient. She was a Muslim religion follower. Then I was trying to perform a physical examination for her. She refused me for touch and she said her religion is not allowed to touch by men (INT 4)." One male nurse added "It is also one barrier of communication. Female Muslim patients are not allowed to touch by the male. This alters our communication with them (INT 7)." Thirty years old nurse continued "If the patients are orthodox they need orthodox nurses, and Muslim also need Muslim nurses. I am working in the operation room now. When patients entered the operation room or undergone surgery; they want to check our neck whether we have a neck loop or not (INT 6).

Being single is another challenge to communicate with the patients. One female nurse said that I have faced many challenges by the patients for marriage questions (INT 2). The other male nurse supports this idea. Once upon a time, I was given care for young female patients. During discharge from the hospital, she asked me to give my phone number as a reason for consultation if something gets worsen from illness. After discharged she told me as she has fallen in deep love with me. This leads to a decrease in my communication with the other single young female patients (INT 3).

\section{Common-related communication barriers}


Common barriers are those barriers that could arise from both sides of the nurse and patients, which can decrease the nurses to patients' communication. The barrier included under this was language difference.

\section{Language differences}

Persons without having common language cannot communicate effectively or properly to express their feeling as those who have a common language.

One of the participants said that the difference in language affects communication with the patients. The patients give other meanings as we told positive things for them (INT 5). The other nurse continued that we have face difficulty in communication with those patients who speak other than Amharic like "Awigna", and "Afan Oromo" language speakers cannot understand us whatever we talk good thing for them (Participant 6). The 50 years old nurses spoken that language difference with the patients also affect our communication for example; we cannot easily communicate with those patients who speak "Agewigna" (INT 1).

\section{Nurse-related barriers}

These are barriers that arise from the nurses, which can inhibit the nurse to patient communication. The barriers under this include workload, shortage of nurses, and lack of communication skills.

\section{Workload}

The presence of workload from the nurses is the potential threat of better care. When nurses carry out activities, more than their capacity they became burnout and unable to satisfy the patients' care needs.

There is a shortage of nurses, as a result, we serve the patients more than our capacity and we feel fatigued, exhausted, and burnout. This damages our communication with the patients (Participant 3). The other participant also continued we faced physical fatigue when we did more than our capacity; this leads to the obstacles of communication (INT 6 and FGD). I cannot give adequate time to communicate with the patients rather I prefer to do the routine activities because of workloads (INT 7).

\section{Shortage of nurses}

The presence of inadequate nurses in the hospitals or few nurses during their shift can damage communication with their patients because of unable to address all demands of the patient very well.

Especially at night shift nurse to patient ratio is one to ten up to twelve (Participant 5 and FGD). A 38 years old nurse said that there are a limited number of nurses compared to the flow of the patients. Therefore, during this time, we prefer to do our routine activities like medication administration, doing the 
nursing process without listening to the patient idea. As a result, our communication with patients is affected (INT 4 and FGD). The other nurse continued we serve more than twenty patients, especially during duty time. If there is a shortage of nurses, we cannot give the required services timely for our clients (Participant 6).

\section{Lack of communication skill}

Communication skill for nurses is very essential to communicate effectively with their patients. Nurses without good communication skills, cannot provide better care for the patients.

One nurse told that some nurses have natural behaviour that cannot shape with training (Participant 2). The other nurse continued we most nurses have lacked the skills to communicate with the patient like the place we select for communication, how to start communication, and are patients understand me or not...is not considered (INT 4). Some nurses cannot fully explain what things are going to do for their patients about care or treatments (INT 6). The other nurse said that I know one nurse she was assigned to work with me together in the pediatric ward. She was having an ethical problem. She made conflict most of the time with the patients. The entire mother knows her ethical problem and they always complained that we are not voluntary if this red nurse gives our children's medication. Therefore, this was a great barrier to communication with the patients (INT 7 and FGD).

\section{Patient-related barriers}

Patient-related barriers are these obstacles that arise directly from the patients that inhibit nurse to patient communication. The barriers included under these were pain and family interference.

\section{Pain}

Pain is a general term that describes uncomfortable sensations in the body. It can change the behaviour of the patients from stable to irritable mood and results in refuses to make contact with their caregiver.

One nurse revealed that the presence of pain decreases the communication between nurses and patients. If the patients are, in the good condition, they have a good facial expression for nurses greeting but if they are in the pain, they cannot respond to our greeting (INT 1). The other participant also said that as the patients suffering from the pain, they are not voluntarily communicating with us (INT 4). One of the participants also continued if the patients get pain they are not voluntary to communicate with the nurses (INT 5 and FGD). The 47 years old nurse said that patients with severe pain cause to disrupt our communication. Patients are not voluntary to give accurate data to us unless we give anti-pain and were reliving from pain (INT 7). 


\section{Family interference}

Conflict in the caring environment is common between care providers and the patients' attendants either intentionally or unintentionally. This is because of the unnecessary interferences of family or attendant with the caring process.

One of the nurses told us that during we give care for the patients the family interferes with our activities. This makes angry for the nurses and leads to conflict with them and finally, communication with the patients inhibited. For example, one day the patient medication was discontinued in around session then the attendant comes and complained that why not give the medication. The nurse responds for the attendant as it was discontinued, finally the attendant fight with the nurse why you discontinued it as it is already prescribed by the physician (INT 5). The other nurse continued that at one-time one-college students come to us because of illness. During this time, we were trying to help her but; her friends come and disturbed us. They said this is not the disease rather she attacks by an evil eye person so, this cannot be treated by modern medicine, and they try to hit one of the nurses with us (INT 7).

\section{Environmental-related barriers}

These barriers are arising directly from the health care setting which caused the barrier of the nurse to patient communication. The barriers included under these were lack of continuous training, lack of medical facilities for the patients, inappropriate and busy environment.

\section{Lack of continuous training on communication}

If nurses do not get continuous training regularly, they cannot update themselves and they may easily subject to tradition as well as lacked basic caring skills.

To increase our communication with the patients we need to have continuous training. But there is no training to enhance the nurse capacity especially on communication skills (INT 4 and FGD). The other nurse continues, we need to have training on communications skill to enhance our communication with the patients (INT 5). Lack of training on communication is the major barrier to communication with the patients so, short training needs to enhance the nurse to patient communication (INT 7 and FGD). The other nurse continued training is not given; even it provided for those who not concerned about it (INT 6).

\section{Lack of medical facilities}

If the hospitals cannot provide the necessary medical equipment or materials for the patients; the patients complained goes to their immediate caregiver or nurses. This is the main cause of the communication barrier. 
All participants said that a lack of medical facilities was a barrier to communication. One female nurse said the hospitals could not provide all necessary medical facilities for the patients like a drug. For example, most societies in this surrounding area used health insurance. We prescribe drugs to the patients but they cannot get the drug inside the hospital rather they pushed to buy out of the hospital or in the private pharmacy. Then the patients complain to us, as they cannot afford to buy the drug. We told the truth as it is not our responsibility and if hospitals can list out the non-available drug and post it to the working unit, we cannot prescribe it. They did not listen to us. This leads to conflict between nurses and patients and decreases communication (INT 1). The other nurse continued that the health institution related issues are affecting our communication. For example, we send the patient to buy the drug out of the hospital then the patient made conflict with us. This is happening because the hospital cannot provide an adequate supply of drugs. This alters our communication with the patients (INT 2). Health insurance is another challenge for communication. The hospital cannot fulfil all the necessary drugs and the patients bought them out of the hospital. After that, they ask us to audit the cost of the drug. We respond to them our duties is to prescribe the drugs not auditing cost. This affects the communication we have with the patients (INT 3). One male nurse also stated that the patient comes to the hospital with their health insurance, and drugs are not available adequately. During this time, the patient is not interested to listen to us whatever we talk about. This challenges our communication with the patients (INT 4). The patients come with their health insurance, and they expect everything inside the hospital. If they did not get the services as they expected they shout towards us. In this condition, our communication with the patients is affected (INT 5 and FGD). Almost all persons use health insurance and the hospital cannot provide all the patient medical facilities like drug supply. If they cannot afford to buy the drug out of the hospitals, their treatment may discontinue and they complain to us why the treatment discontinued. In this time, we lead to an unnecessary verbal fight with the patients (INT 6). Patients come with health insurance for free services but the hospital cannot provide all the services like drug supply; when they ordered to buy out of the hospital the make conflict with us (INT 7).

\section{Inappropriate environment}

Unsafe caring environments are among the obstacles of the nurse to patient communication. Unattractive health care environments can hinder the interaction between nurses and patients.

Participants reported that poor sanitation of the room also affects the communication between nurses and patients (INT 4, 7 and FGD).

\section{Busy environment}

A busy environment or the crowdedness of the health care environment is can inhibit nurse to patient communication. 
One of the nurses said that the place of the hospital is nearing the road especially the emergency ward, the sound of the cars also affects us (INT 5). The other nurse also continued his idea for example when I enter the ward to care for my patient I saw the persons who make crowded rooms at that time I prefer to leave the room; because the environment was not suitable for me to communicate with my patient (INT 7).

\section{Discussion}

The main purpose of this study was to explore the experience of Perceived communication barriers include; common-related communication barriers; nurse-related, patient-related, and environmental-related barriers.

Age, gender, religion, marital status, language difference, workload, shortage of nurses, lack of communication skills, pain, family interference, lack of continuous training on communication skills, lack of medical facilities, busy environment and inappropriate environment were perceived communication barriers.

Participants in this study indicated that age was the barrier to communication. This finding is similar to studies conducted in Iran and Ghana showed that age was the communication barrier between nurses and patients (17-19)

This finding revealed that gender was the main perceived communication barriers. This is because of having sexual desire in the opposite gender. Studies were done in India, and Iran supported this idea (5, $18,20)$.

From this experience of nurses, religion was another perceived barrier of communication. The reason is Muslim religion followers have the court of sharia which restricts that females body should not be exposed and not touch by males other than their husbands. This idea is similar to Evidence Nigeria, and Ghana showed that religion was negatively affected nurses' communication with patients $(4,17,21)$.

The Marital-status of the nurses and patients was found to be the barrier of communication. This finding has supported a study done in India showed that the marital status of nurses affects communication. Single female nurses had difficulty in communicating in a friendly manner with male patients (20).

Language difference was perceived common-related communication barrier reported by nurses. This is because; the presence of multilingual people in Ethiopia can be a barrier to communication. This result is similar to the studies done in Saudi Arabia in which nurses were reported that they faced difficulty in dealing with patients because of language differences (22). Another study in Saudi Arabia also supported this finding in which some of the communication practices rely on non-verbal methods due to a lack of a common language which often results in misinterpreted of the meaning of the communication (23).

In this study, being loaded in activities or workload was the barrier of the nurse to patient communication. Because when nurses working beyond their capacity they become exhausted and burnout as a result they 
cannot easily interact or communicate with their patients. This result is in line with a study conducted in Ghana; in which nurses were agreed that overwork as the barrier to communication (17). This finding also supported by the studies done in two different areas of Iran indicated that workload was the barrier of communication between nurses and patients $(18,19)$. It also supported by a study in Saudi Arabia in which nurses were agreed that heavy workload as a barrier of the nurse to patient communication (4).

The shortage of nurses was a barrier to communication. The main reason is in this area the nurse to patient ratio reaches one to twenty or more compared to in the state of California the maximum nurse to patient ratio is one to six (24). This finding is similar to a study done in Ghana in which nurses were agreed that the shortage of nurses was the perceived barrier of communication (17). Other Studies in two different areas of Nigeria also supports that inadequate or shortage of nurses was affect the nurse to patient communication $(21,25)$.

In this study lack of communication skills was the communication barriers. The finding is similar to the study done in Singapore in which nurses were less skilled to engage in communication (26).

This study shows the presence of pain was a barrier to communication. The main reason that patients seek to visit health institutions is the presence of pain. If pain cannot manage properly, the patients do not have the interest to communicate with their caregiver. This study is supported by a study done in Ghana that indicated that Pain was the major patient-related barriers to communication (17).

In this study, family interference is the other barrier of the nurse to patient communication. It is due to the interference of family members in the patient caring process unnecessarily, the nurse preferred to leave the patient. This is aligned with the study done in Saudi Arabia, and a qualitative study in Ghana indicated that family interference was the barrier to Communication $(4,27)$.

In this study, the majority of the participants were agreed that the lack of continuous training in communication skills was a barrier to communication. This is because nurses without adequate continuous training on communication skills can easily vulnerable to the communication barrier. This result is similar to the study done in Saudi Arabia indicated that there was general agreement among the nurses that lack of continuous training in communication skills was seen as a communication barrier between nurses and patients (4).

Findings showed that the lack of medical facilities was the barrier of the nurse to patient communication. Because patients come to the hospitals to get adequate services, if not this, patients make conflict with the frontline caregiver or nurses. This result is similar to the study done in Egypt showed that there were inadequate facilities that affect patients' communication with nurses(28). It also supported by the study done in Saudi Arabia nurses were showed their agreement as a barrier of communication (4).

In this study, findings showed that a busy environment is one of the environmental-related communication barriers. Unsafe environments make boredom relationships between nurses and patients as a result they cannot create comfortable conversations on both sides. This is supported by the studies 
done in Iran, Ghana, and Egypt showed that busy or crowded rooms were the main environment-related communication barrier $(17,28,29)$.

Generally, the finding revealed that there are perceived communication barriers including sociodemographic characteristics, common-related, nurse-related, patient-related, and environmental or hospital-related barriers, which affect nurse to patient communication. The result of this study has multidimensional implications. It can be used for nurses to deal with and overcome communication barriers. Dealing with the communication barriers mean also dealing with the problems of the patients so that the patients can get better care from their caregiver. As the barrier going to be minimizing or decreasing, the hospitals can be attractive for patients, safe for healing, increasing patient satisfaction, decrease hospital stay, and helps to minimize health care costs. Finally, this finding can be used as a baseline for further research.

\section{Limitations of the study}

This study was focused on the experiences of nurses only; the experience of patients on the communication barrier was not explored.

\section{Conclusion}

Nurses, patients, and the environments are the main perceived communication barriers as indicated in both in-depth interviews and focused group discussion. Lack of medical facilities or access is the main barrier of the nurse to patient communication, which needs great attention of the stakeholders. Nurse professionals need to have good communication skills to solve or overcome the problem of patients; and must communicate effectively to perform their roles as educators, managers, decision-makers, client advocators, problem solvers, and caregivers. To enhance communication with the patients; nurses and other stakeholders like the ministry of health, health bureaus, and hospital authorities need to recognize the communication barriers. Giving awareness on the communication barriers for the nurses helps to minimize the barriers and improve the nurses to patients' communication.

\section{Declarations}

We declared that all methods were carried out per relevant guidelines and regulations of the qualitative research and the submission has been prepared based on BMC Nursing journal guidelines.

\section{Ethics approval and consent to participate:}

Ethical approval was obtained from Bahir Dar University ethical approval committee. A formal permission letter was communicated at each level. The participants were well informed about the purpose of the study and information was collected after oral consent from nurses working in hospitals. 


\section{Consent for publication:}

Not applicable.

\section{Availability of data and material:}

The data are available from the corresponding author and will be accessed as needed.

\section{Competing interests:}

All authors declared that they have no competing interests and no organizations have financial or other conflicts of interest in this paper.

\section{Funding:}

Currently, there are no Funding for this research, all the costs were covered by the authors.

\section{Authors' contributions:}

Corresponding author: design the study, analyzed the data, interpretation of the data, and wrote the manuscript and all the authors have almost equal contributions for this study. Finally, all the authors read and approved the manuscript.

\section{Acknowledgements:}

We would like to acknowledge all the data collectors and study participants for their contributions to the success of our work.

\section{References}

1. Bramhall Elaine. Effective communication skills in nursing practice. Nurs Stand. 2014 Dec 9;29(14):53-9. PubMed PMID: 25467362. Epub 2014/12/04. eng.

2. Kourkouta Lambrini, Papathanasiou loanna V. Communication in nursing practice. Mater Sociomed. 2014 Feb;26(1):65-7. PubMed PMID: 24757408. Pubmed Central PMCID: PMC3990376. Epub 2014/04/24. eng.

3. Wright Ros. Effective communication skills for the "caring" nurse. The Great Teachers: Tertiary Place. 2012. 
4. Albagawi Bander S, Jones Linda K. Quantitative exploration of the barriers and facilitators to nursepatient communication in Saudia Arabia. Journal of Hospital Administration. 2017;6(1):16.

5. Andriyanto. Communication barrier between nurse and patient at the hospital: A systematic review. Journal of Health Policy and Management. 2019;4(2):105-10.

6. Rani K Usha. Communication barriers. 2016.

7. Bridges et al. Capacity for care: Meta-ethnography of acute care nurses' experiences of the nursepatient relationship. Journal of Advanced Nursing. 2013;69(4):760-72.

8. Hemsley Bronwyn, Balandin Susan, Worrall Linda. Nursing the patient with complex communication needs: Time as a barrier and a facilitator to successful communication in hospital. Journal of advanced nursing. 2012;68(1):116-26.

9. Norouzinia Roohangiz, Aghabarari Maryam, Shiri Maryam, Karimi Mehrdad, Samami Elham. Communication barriers perceived by nurses and patients. Global journal health science. 2015 Sep 28;8(6):65-74. PubMed PMID: 26755475. Pubmed Central PMCID: PMC4954910. Epub 2016/01/13. eng.

10. Hunt Steve, Hunt Elena. Barriers to the practice of rural and remote nursing in Canada. European Scientific Journal. 2016;12(36):7857-81.

11. Haugan Gørill. Nurse-patient interaction is a resource for hope, meaning in life and selftranscendence in nursing home patients. Scandinavian Journal of Caring Sciences. 2014;28(1):7488.

12. Lasiter Sue. "The button" initiating the patient-nurse interaction. Clinical nursing research. 2014;23(2):188-200.

13. Rezende Laura Cristhiane Mendonça, Costa Kátia Neyla De Freitas Macedo, Kaisy Pereira Martins Tatiana, Ferreira Da Costa Sérgio Ribeiro Dos Santos, Leite Kamila Nethielly Souza. Therapeutic communication between nurses and patients in pre-operative during an admission in a medical surgical unit. surgery. 2013;4:5.

14. Vida Maame Kissiwaa Amoah et al. A qualitative assessment of perceived barriers to effective therapeutic communication among nurses and patients. BMC nursing. 2019;18(1):4.

15. Saunders Benjamin, Julius Sim, Tom Kingstone, Shula Baker, Jackie Waterfield, Bernadette Bartlam, et al. Saturation in qualitative research: Exploring its conceptualization and operationalization. Quality \& quantity. 2018;52(4):1893-907.

16. Clarke Victoria, Virginia Braun, Nikki Hayfield. Thematic analysis. Qualitative psychology: A practical guide to research methods. 2015:222-48.

17. Amoah Vida Maame Kissiwaa, Reindolf Anokye, Dorothy Serwaa Boakye, Enoch Acheampong, Amy Budu-Ainooson, Emelia Okyere, et al. A qualitative assessment of perceived barriers to effective therapeutic communication among nurses and patients. BMC nursing. 2019;18(1):4.

18. Anoosheh M, Zarkhah S, Faghihzadeh S, Vaismoradi M. Nurse-patient communication barriers in Iranian nursing. Int Nurs Rev. 2009;56(2):243-9. 
19. Jahromi Marzieh Kargar, Ramezanli Somayeh. Evaluation of barriers contributing in the demonstration of effective nurse-patient communication in educational hospitals of Jahrom, 2014. Global journal of health science. 2014;6(6):54.

20. Zamanzadeh V, Rassouli M, Abbaszadeh a, Nikanfar a, Alavi-Majd H, Ghahramanian A. Factors influencing communication between the patients with cancer and their nurses in oncology wards. Indian journal of palliative care. 2014;20(1):12.

21. Arungwa Oluwatosin Taiwo. Effect of communication on nurse-patient relationship in national orthopaedic hospital, Igbbobi, Lagos. West African journal of nursing. 2014;25(2).

22. Al-Harasis Sameer. Impact of the language barrier on quality of nursing care at armed forces hospitals, Taif, Saudi Arabia. Middle East Journal of Nursing. 2013;101(665):1-8.

23. Alshammari Mukhlid, Duff Jed, Guilhermino Michelle. Barriers to nurse-patient communication in Saudi Arabia: An integrative review. BMC nursing. 2019;18(1):61.

24. Lippincott. The importance of the optimal nurse-to-patient ratio Nov 102016.

25. Olufunke Obayori Christianah, Omolola Oluwakorede. Peplau's theory of psychodynamic nursing and nurse-patient interaction: A literature review.

26. Tay Li Hui, Hegney Desley, Ang Emily. Factors affecting effective communication between registered nurses and adult cancer patients in an inpatient setting: A systematic review. International Journal of Evidence-Based Healthcare. 2011;9(2):151-64.

27. Maame Kissiwaa Amoah Vida, Anokye Reindolf, Boakye Dorothy Serwaa, Gyamfı Naomi. Perceived barriers to effective therapeutic communication among nurses and patients at Kumasi south hospital. Cogent Medicine. 2018;5(1):1459341.

28. Ibrahim Azza Fathi, Tawfik Faiza Mohammed, Akel Dalal T. Nurse communication in health education: Patients' perspective. Clinical Nursing Studies. 2015;3(4):94-102.

29. Bakhitari Soheyla, Mohammadzadeh Shahla, Moshtagh Zahra. Obstacles in nurse - elderly relationship from the patients' points of view. 2009. 